\title{
Elevated levels of cerebrospinal fluid $\alpha$-synuclein oligomers in healthy asymptomatic LRRK2 mutation carriers
}

\section{Jan O. Aasly ${ }^{1,2}$ *, Krisztina K. Johansen ${ }^{1}$, Gunnar Brønstad ${ }^{2}$, Bjørg J. Warø ${ }^{1,2}$, Nour K. Majbour ${ }^{3}$, Shiji Varghese ${ }^{3}$, Fatimah Alzahmi ${ }^{3}$, Katerina E. Paleologou ${ }^{4}$, Dena A. M. Amer ${ }^{3}$, Abdulmonem Al-Hayani ${ }^{5}$ and Omar M. A. El-Agnaf ${ }^{3,6 *}$}

\author{
1 Department of Neuroscience, Norwegian University of Science and Technology (NTNU), Trondheim, Norway \\ 2 Department of Neurology, St. Olav's Hospital, University Hospital of Trondheim, Trondheim, Norway \\ ${ }^{3}$ Department of Biochemistry, College of Medicine and Health Sciences, United Arab Emirates University, Al Ain, United Arab Emirates \\ ${ }^{4}$ Department of Molecular Biology and Genetics, Democritus University of Thrace, Alexandroupolis, Greece \\ ${ }^{5}$ Department of Anatomy, Faculty of Medicine, King Abdulaziz University, Jeddah, Saudi Arabia \\ ${ }^{6}$ Faculty of Medicine, King Abdulaziz University, Jeddah, Saudi Arabia
}

\author{
Edited by: \\ Manuel Menéndez-González, \\ Hospital Álvarez-Buylla, Spain \\ Reviewed by: \\ Catarina Oliveira, University of \\ Coimbra, Portugal \\ Kenjiro Ono, Kanazawa University \\ Graduate School of Medical \\ Science, Japan \\ *Correspondence: \\ Jan O. Aasly, Department of \\ Neurology, St. Olav's Hospital, \\ University Hospital of Trondheim, \\ Edvard Grieg's Gate 8, 7006 \\ Trondheim, Norway \\ e-mail: Jan.Aasly@ntnu.no; \\ Omar M. A. El-Agnaf, Department \\ of Biochemistry, College of \\ Medicine and Health Sciences, \\ United Arab Emirates University, \\ Tawam Medical Campus, Khalifa \\ Street, Al-Ain, United Arab Emirates \\ e-mail: o.elagnaf@uaeu.ac.ae
}

Mutations in the leucine-rich repeat kinase 2 gene are the most common cause of autosomal dominant Parkinson's disease (PD). To assess the cerebrospinal fluid (CSF) levels of $\alpha$-synuclein oligomers in symptomatic and asymptomatic leucine-rich repeat kinase 2 mutation carriers, we used enzyme-linked immunosorbent assays (ELISA) to investigate total and oligomeric forms of $\alpha$-synuclein in CSF samples. The CSF samples were collected from 33 Norwegian individuals with leucine-rich repeat kinase 2 mutations: 13 patients were clinically diagnosed with PD and 20 patients were healthy, asymptomatic leucine-rich repeat kinase 2 mutation carriers. We also included 35 patients with sporadic PD (sPD) and 42 age-matched healthy controls. Levels of CSF $\alpha$-synuclein oligomers were significantly elevated in healthy asymptomatic individuals carrying leucine-rich repeat kinase 2 mutations $(n=20 ; P<0.0079)$ and in SPD group $(n=35 ; P<0.003)$ relative to healthy controls. Increased $\alpha$-synuclein oligomers in asymptomatic leucine-rich repeat kinase 2 mutation carriers showed a sensitivity of $63.0 \%$ and a specificity of $74.0 \%$, with an area under the curve of 0.66 , and a sensitivity of $65.0 \%$ and a specificity of $83.0 \%$, with an area under the curve of 0.74 for SPD cases. An inverse correlation between CSF levels of $\alpha$-synuclein oligomers and disease severity and duration was observed. Our study suggests that quantification of $\alpha$-synuclein oligomers in CSF has potential value as a tool for PD diagnosis and presymptomatic screening of high-risk individuals.

Keywords: Parkinson's disease, LRRK2 mutation carriers, CSF, biomarkers, alpha-synuclien

\section{INTRODUCTION}

Parkinson's disease (PD) is the most common age-related movement disorder and the second most common neurodegenerative disorder after Alzheimer's disease. The earliest clinical features of PD are typically retrospective and not specific, and they may include depression, hyposmia, constipation, and sleep disorders. At least $70 \%$ of the neurons in the substantia nigra (SN) are lost prior to the appearance of any major motor symptoms (Schapira, 1999). The main motor symptoms, including resting tremor, rigidity, bradykinesia, and postural instability, are collectively known as parkinsonism. Currently, PD is clinically diagnosed and clinical trials of disease-modifying drugs are initiated only after most of the vulnerable dopaminergic neurons in the SN have already been lost. Individuals at risk for PD with less complete loss of dopaminergic neurons may be more responsive to and benefit most from neuroprotective therapies. Therefore, identifying biomarkers for early diagnosis may facilitate the development of novel treatments designed to slow disease progression. Furthermore, such findings may help to elucidate the pathophysiology of
PD. Most PD cases are sporadic (sPD) (i.e., idiopathic; attributed to unknown causes). However, some atypical cases involve genetic susceptibility (Singleton et al., 2013). Over the last two decades, several genetic causes of PD have been identified. At present, $5-10 \%$ of all PD cases can be traced to a known genetic cause that is either monogenic or related to a combination of susceptibility factors (Singleton et al., 2013). Mutations in the gene encoding $\alpha$-synuclein ( $\alpha$-syn) (SNCA) were the first genetic factors to be linked to familial PD (Polymeropoulos et al., 1997; Krüger et al., 1998; Singleton et al., 2003). Although SNCA mutations rarely cause late-onset familial PD, SNCA is still of great importance to PD etiology, as abnormal aggregation of $\alpha$-syn in the brain is also found in neuropathological lesions (Lewy bodies (LBs); Spillantini et al., 1997). However, it has been previously shown that $\alpha$-syn is normally released by neuronal cells and present in the cerebrospinal fluid (CSF) and peripheral plasma (El-Agnaf et al., 2003) Recent studies have demonstrated that oligomeric forms of $\alpha$-syn are neurotoxic species in vitro and in vivo, whereas amyloid fibrils may not be directly toxic (Winner et al., 2011). Gene 
mutations in leucine-rich repeat kinase 2 (LRRK2) are the second most common cause of autosomal dominant PD and cause 2$5 \%$ of familial PD. The most common point mutation, G2019S, has been shown to be involved in 5-6\% of autosomal dominant PD cases (Di Fonzo et al., 2005; Nichols et al., 2005; Dächsel and Farrer, 2010) and 1-2\% of sPD cases (Gilks et al., 2005). Patients with late-onset monogenic forms of PD may demonstrate subtle signs or symptoms several years before they suffer from any motor symptoms (Sossi et al., 2010; Johansen et al., 2011; RuizMartínez et al., 2011). Similarly, recent positron emission tomography (PET) studies have confirmed dopaminergic dysfunction in asymptomatic LRRK2 mutation carriers (Nandhagopal et al., 2008). Therefore, these carriers are an ideal population for identifying novel biomarkers for the early diagnosis of PD. We and other groups recently reported elevated levels of $\alpha$-syn oligomers (o- $\alpha$-syn) and an increased o- $\alpha$-syn/total- $\alpha$-syn ( $t-\alpha$-syn) ratio in CSF from PD patients relative to controls (Tokuda et al., 2010; Park et al., 2011; Sierks et al., 2011; Parnetti et al., 2014a,b). These findings suggest that CSF $\alpha$-syn oligomers could be a potentially useful biomarker for diagnosis and possible early detection of PD. We therefore explored the potential use of o- $\alpha$-syn as an early biomarker for PD in CSF from asymptomatic LRRK2 mutation carriers and symptomatic LRRK2 PD patients relative to SPD patients and healthy age-matched controls.

\section{MATERIALS AND METHODS \\ PATIENT POPULATION AND CLINICAL METHODS}

In total, 33 Norwegian individuals from 12 different families with mutant LRRK2 were assessed in this study. Thirteen patients were clinically diagnosed with PD and 20 patients were healthy, asymptomatic LRRK2 mutation carriers. These families have been extensively described in previous report (Aasly et al., 2005, 2010; Johansen et al., 2010). In addition, 35 patients with sPD and 42 age-matched healthy controls were also recruited for this study from St. Olav's Hospital at the University Hospital of Trondheim in Norway. Parkinson's disease was diagnosed according to established diagnostic criteria (Gelb vs. UK Parkinson's Disease Society). Disease severity was defined according to the Hoehn and Yahr scale (H\&Y). All patients with sPD were screened and tested negative for known $L R R K 2$ mutations. Patients with age at onset $\leq 50$ years also tested negative for known pathogenic mutations in Parkin and PINK1. All family members were screened for clinical signs of PD and found to be asymptomatic, although a few had mild pre-motor signs with an increased Unified Parkinson's Disease Rating Scale (UPDRS) score (Johansen et al., 2011). The LRRK2-mutant PD patients were on levodopa, and some were taking other dopamine agonists and monoamine oxidase- $\mathrm{B}$ (MAO-B) inhibitors. The mean levodopa-equivalent dose in the LRRK2-mutant PD group varied between 300 and $1800 \mathrm{mg}$, with a mean of $580 \pm 422 \mathrm{mg}$, and the mean levodopa-equivalent dose in the sPD group was 300 to $1500 \mathrm{mg}$, with a mean of $628 \pm$ $387 \mathrm{mg}$.

All individuals underwent lumbar puncture between 08:00 am and 10:00 am following overnight fasting. A small sample of CSF was sent for routine analysis, and then 18 to $22 \mathrm{ml}$ was sampled and frozen in 15 aliquots of $1.2-1.5 \mathrm{ml}$ each within $15 \mathrm{~min}$ of completion of the lumbar puncture. The aliquots were stored at $-80^{\circ} \mathrm{C}$ until further analysis. All patients gave signed, informed consent, and the study was approved by the Regional Committee for Medical and Health Research Ethics.

\section{SIZE EXCLUSION CHROMATOGRAPHY (SEC) FOR SEPARATING $\alpha$-syn MONOMERS AND OLIGOMERS}

Size Exclusion Chromatography was carried out using an AKTA FPLC system (GE Healthcare-Sweden) and a superdex 200 column at $4^{\circ} \mathrm{C}$. Concentrated $0.5 \mathrm{ml}$ of CSF were loaded onto the column and eluted with PBS $(\mathrm{pH} 7.4)$ at a flow rate of $0.1 \mathrm{ml} / \mathrm{min}(0.5 \mathrm{ml} /$ fraction $)$. The elution of $\alpha$-syn was monitored at absorbance wavelengths of $215 \mathrm{~nm}$. Fractions of $1 \mathrm{ml}$ were collected, concentrated to $100 \mu \mathrm{l}$ using a speed vac, and analyzed by the western blotting for the presence of $\alpha$-syn. To determine the elution time of monomeric and oligomeric $\alpha$-syn, molecular weight standards (Thyroglobulin $669 \mathrm{kDa}$, ferritin $440 \mathrm{kDa}$, aldolase $171 \mathrm{kDa}$, abmumin $68 \mathrm{kDa}$ and chymotrypsinogen $\mathrm{A}$ $25 \mathrm{kDa})$, fresh $\alpha$-syn solution and aged $\alpha$-syn solution were co-injected into the column and eluted at the same conditions mentioned above.

\section{SODIUM DODECYL SULFATE-POLYACRYLAMIDE GEL ELECTROPHORESIS (SDS-NUPAGE) AND IMMUNOBLOTTING}

The CSF fractions were separated on NuPAGE Bis-Tris 4-12\%, $1 \mathrm{~mm}$ gels (Invitrogen Ltd., Paisley, UK) and then transferred to nitrocellulose membranes $(0.45 \mu \mathrm{m})$ at $30 \mathrm{~V}, 125 \mathrm{~mA}$ for $45 \mathrm{~min}$ (Invitrogen Ltd., Paisley, UK). Membranes were boiled for $5 \mathrm{~min}$ in PBS then blocked for $1 \mathrm{~h}$ with $5 \%$ marvel dried skimmed milk and dissolved in PBS-Tween 20 (0.05\%) (PBST). The membranes were probed overnight at $4^{\circ} \mathrm{C}$ anti- $\alpha$-syn (211) mouse monoclonal antibody to $\alpha$-syn (aa 121-125). The membranes were washed several times with PBST followed by incubation with horseradish peroxidase (HRP)-conjugated goat anti-mouse (Dako Ltd., Ely, UK), for $60 \mathrm{~min}$. The membranes were again washed several times with PBST. The protein bands were visualized using ECL reagents (Pierce, USA) as described by the manufacturer.

\section{IMMUNOASSAYS FOR TOTAL AND OLIGOMERIC $\alpha$-SYNUCLEIN IN CSF}

A sandwich enzyme-linked immunosorbent assay (ELISA) methods were employed to measure total or oligomeric $\alpha$-syn levels in CSF samples as described previously (Tokuda et al., 2010).

\section{STATISTICAL ANALYSIS}

Differences between groups were compared using a MannWhitney $\mathrm{U}$ test. Significance was defined as $P<0.05$. Correlational analysis was conducted by Pearson simple correlation. The receiver operating characteristic (ROC) was analyzed to assess the most appropriate cut-off values for the level of CSF $\alpha$-syn oligomer and the oligomers/total- $\alpha$-syn ratio in the CSF to distinguish between groups. All analyses were conducted using GraphPad Prism software (GraphPad Prism Version 4.0, GraphPad software, San Diego, CA).

\section{RESULTS}

\section{PATIENT POPULATION AND DEMOGRAPHICS}

In total, 33 Norwegian individuals from 12 different LRRK2 families were investigated in the present study. Thirteen individuals 
with LRRK2 point mutations had developed symptomatic PD, including 11 males who were carrying the most common LRRK2 point mutation, G2019S, and two females who were carrying a different LRRK2 point mutation, N1437H. The 13 individuals had a mean age of 64.0 years \pm 13.3 years. In contrast, 20 individuals were healthy asymptomatic LRRK2 mutation carriers [G2019S $(n=16)$ and $\mathrm{N} 1437 \mathrm{H}(n=4)]$. These 20 individuals had a mean age of 55.4 years \pm 15 years. None of the healthy asymptomatic LRRK2 mutation carriers (LRRK2-H) had any complaints of a movement disorder. Some were receiving medication for diabetes mellitus, mild hypertension, and other minor health problems. In addition, 35 patients with sPD and 42 age-matched healthy controls were also included in this study. No significant difference was noticed in disease duration between symptomatic PD patients with LRRK2 mutations (LRRK2-PD) and sPD patients. Moreover, there was no difference between the groups with regard to CSF levels of leukocytes or total protein, albumin, and glucose levels, including plasma glucose levels. Controling for age and gender did not significantly alter the results in any case. A summary of the patient population employed in the present study and the respective demographic details are shown in Table 1.

\section{LEVELS OF TOTAL $\alpha$ Syn (t- $\alpha$ syn) IN CSF SAMPLES}

To measure the total $\alpha$-syn (t- $\alpha$-syn) in CSF samples, we recently optimized our original $\alpha$-syn ELISA protocol using a chemiluminescence-based read-out arm for HRP-labeled antibody detection (Tokuda et al., 2010). We demonstrated that our optimized protocol yielded excellent performance with regard to both specificity and sensitivity. Using this system, an increase of approximately 100 -fold in the detection of recombinant $\alpha$-syn was recorded, ranging from 0.010 to $>500 \mathrm{ng} / \mathrm{ml}$ (Tokuda et al., 2010).

As illustrated in Figure 1, the concentration of t- $\alpha$-syn varied considerably among the four studied groups, although the difference was not statistically significant. Lower mean concentrations of CSF $t$ - $\alpha$-syn were observed in patients with $\mathrm{sPD}$ (mean $\pm \mathrm{SEM}=22.81 \pm 4.198 \mathrm{ng} / \mathrm{ml}, n=35$ ), LRRK2-PD (mean \pm SEM $=20.54 \pm 3.139 \mathrm{ng} / \mathrm{ml}, n=13)$, and LRRK2-H (mean \pm SEM $=17.84 \pm 2.569 \mathrm{ng} / \mathrm{ml}$, $n=20$ ) than in age-matched controls (mean \pm SEM $=$ $24.74 \pm 4.470 \mathrm{ng} / \mathrm{ml}, n=42)(P=0.7001$, Mann-Whitney U-test).

\section{CSF $\alpha$ syn OLIGOMERS (0- $\alpha$ syn) LEVELS}

After measuring the total $\alpha$-syn in CSF samples from our cohort, we then measured the levels of CSF $\alpha$-syn oligomers (o$\alpha$-syn) in the same samples. For such measurements, we used an ELISA system for $\alpha$-syn oligomers that specifically detects soluble oligomers without detecting monomeric forms of $\alpha$-syn (El-Agnaf et al., 2006). Because $\alpha$-syn oligomers represent a small fraction of the total $\alpha$-syn in CSF, for this assay we also used a chemiluminescence-based read-out arm to allow detection of low levels of CSF $\alpha$-syn oligomers. The ELISA protocol was based on a conventional sandwich system in which mAb 211 was used to capture $\alpha$-syn, followed by detection with a biotinylated form of 211. Subsequently, the biotinylated mAb was detected with ExtrAvidin-HRP, followed by a chemiluminescent substrate. In this assay, no signal is detected for monomeric $\alpha$-syn, as the capture mAb occupies the only available antibody binding site on the protein. In contrast, multiple mAb binding sites are available in the case of oligomeric forms of $\alpha$ synuclein, thus permitting both capture and detection (El-Agnaf et al., 2006). This assay has been extensively characterized and yields excellent performance in both specificity and sensitivity (Tokuda et al., 2010). In order to investigate the size of $\alpha$-syn oligomers detected by our ELISA in CSF, recently we used SEC to fractionate fresh CSF samples from those PD patients that gave a robust signal in our ELISA. The western blot revealed immunoreactive material with an elution peak in SEC fractions corresponding to $\mathrm{MW}$ of 70 and $170 \mathrm{kDa}$ which belong to $\alpha$-syn monomers and dimmers respectively. However, much of the immunereactive material was eluted at the void volume, which indicated a MW of $>670 \mathrm{kDa}$ (Figure 2A). These SEC fractionation results support the notion that the CSF from PD patients mainly contain HMW $\alpha$-syn oligomers, since most of the protein material detected by the ELISA has a MW >760 kDa. Experiments are in progress in our laboratory to further characterize and analyze the structure and nature of the oligomeric species of $\alpha$-syn and to discern any modifications of $\alpha$-syn detected by the ELISA (i.e., nitrated, phosphorylated, etc.). This information will be useful in order to improve both the sensitivity and selectivity for $\alpha$-syn protein species in our future ELISA variants.

A scatter plot representing the CSF levels of o- $\alpha$-syn for each patient in the four groups is shown in Figure 2B; the level of $\mathrm{O}-\alpha$-syn is given as the chemiluminescence signal intensity

Table 1 | Details of patient population employed in the present study and the demographics.

\begin{tabular}{lccc}
\hline Groups & $\begin{array}{c}\text { Symptomatic PD due } \\
\text { to LRRK2 mutations }\end{array}$ & $\begin{array}{c}\text { Asymptomatic LRRK2 } \\
\text { mutation carriers }\end{array}$ & $\begin{array}{c}\text { Sporadic PD } \\
\text { controls }\end{array}$ \\
\hline Number of individuals & 13 & 20 & 42 \\
Gender (M/F) & $11 / 2$ & $11 / 9$ & 35 \\
Age range (y) & $43-87$ & $26-76$ & $23 / 2$ \\
Mean Age (y) & $64 \pm 13.3$ & $55.4 \pm 15$ & $38-71$ \\
Levodopa equivalents & $580 \pm 422 \mathrm{mg}$ & $\mathrm{NA}$ & $54 \pm 15$ \\
H-Y grade & $2.7 \pm 0.7$ & $\mathrm{NA}$ & $628 \pm 387 \mathrm{mg}$ \\
Disease duration (months) & $24-330$ & $\mathrm{NA}$ & $2.28 \pm 0.6$ \\
\hline
\end{tabular}

$P D=$ Parkinson's disease $; y=$ years $M=$ male $;=$ female $; H$-Y grade $=$ Hoehn-Yahr grade. 


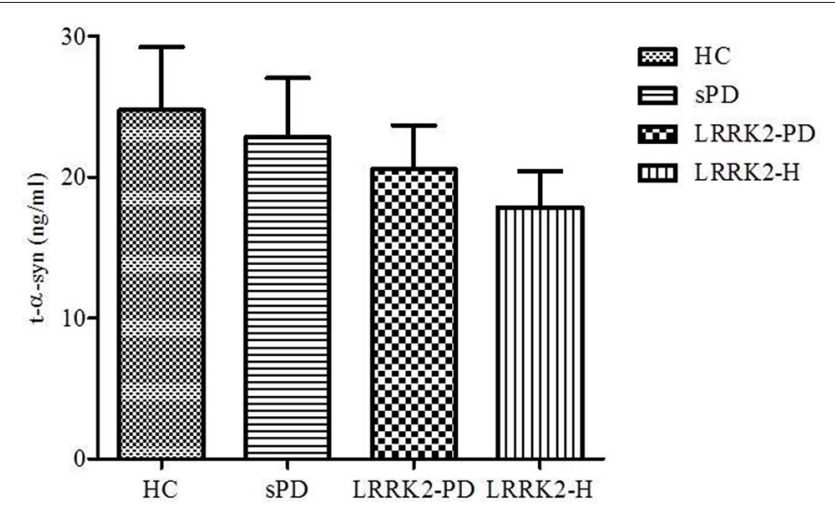

FIGURE 1 | Levels of $\mathrm{t}-\alpha$-syn (in $\mathrm{ng} / \mathrm{ml}$ ) in CSF from healthy controls (HC), sporadic PD patients (sPD), symptomatic PD patients with LRRK2 mutations (LRRK2-PD), and healthy asymptomatic LRRK2 mutation carriers (LRRK2-H). Values are expressed as the mean \pm standard deviation.

(relative luminescence units/second $[\mathrm{RLU} / \mathrm{s}]$ ). The level of CSF o- $\alpha$-syn was significantly higher by 4 -fold in the sPD group (mean $\pm \mathrm{SEM}=80,186 \pm 23,861, n=35$ ) relative to the healthy age-matched control group (mean $\pm \mathrm{SEM}=17,117 \pm$ 2943, $n=42)(P<0.01$, Mann-Whitney U test $)$. Significantly higher levels of CSF o- $\alpha$-syn (approximately 2-fold higher) were also detected in LRRK2-H (mean \pm SEM = 38,754 $\pm 12,514$, $n=20$ ) relative to the control group (mean \pm SEM $=17,117 \pm$ 2,943, $n=42)(P<0.01$, Mann-Whitney U test). Unexpectedly, LRRK2-PD patients (mean $\pm \mathrm{SEM}=24,510 \pm 7,161, n=13$ ) did not show any significant difference in the level of o- $\alpha$-syn in the CSF relative to the healthy control group $(P=0.1910$, Mann-Whitney $U$ test), possibly because of the low number of individuals in this group ( $n=13$ vs. $n=42)$. Furthermore, we observed a significant inverse correlation (Spearman $r=-0.49$, $P<0.05)$ between the severity of the disease and the level of o$\alpha$-syn in the CSF of sPD patients using H\&Y grading (Table 2). However, no significant correlation (Spearman $r=-0.5391, P$ $=0.09$ ) between $H \& Y$ grading and the levels of $o-\alpha$-syn in the CSF of LRRK2-PD patients was observed (Table 2). Interestingly, when SPD and LRRK2-PD cases were combined together, more significant and stronger inverse correlation was noted (Spearman $r=-0.6, P=0.0004$, Table 2). Whereas, CSF t- $\alpha$-syn levels did not correlate with $H \& Y$ levels. In parallel, statistically significant inverse correlation between CSF o- $\alpha$-syn and disease duration was also observed in sPD group (Spearman $r=-0.45, P<0.05$ ), but CSF o- $\alpha$-syn did not correlate with disease duration in LRRK2$\mathrm{PD}$ group. Whereas, we observed significant negative correlation between CSF o- $\alpha$-syn levels and disease duration when the sPD and LRRK2-PD groups were combined together (Spearman $r=$ $-0.5, P=0.002$, testable 2 ). In contrast, CSF o- $\alpha$-syn levels did not correlate with UPDRS scores within any of the groups (data not shown). In addition, no correlation was observed between the level of $o-\alpha$-syn in the CSF and the age of the patients (data not shown).

The measurement of both total and oligomeric $\alpha$-syn in $\mathrm{RLU} / \mathrm{s}$ allowed us to calculate the ratio of $o-\alpha$-syn to $\mathrm{t}-\alpha$-syn (o- $\alpha$-syn/t- $\alpha$-syn ratio [\%]) in the CSF of each patient, as shown in Figure 3. This ratio was found to be significantly higher in the sPD group (mean $\pm \mathrm{SEM}=71.59 \pm 12.76$,
A

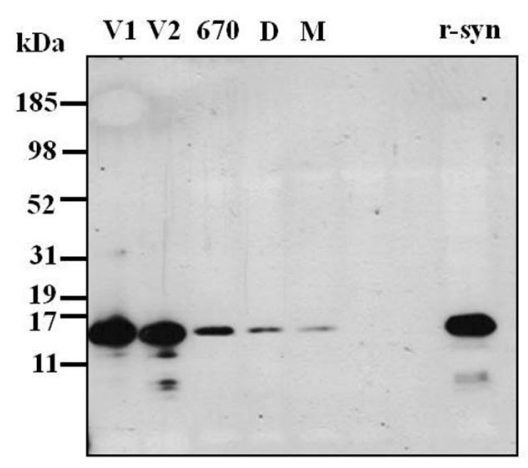

B

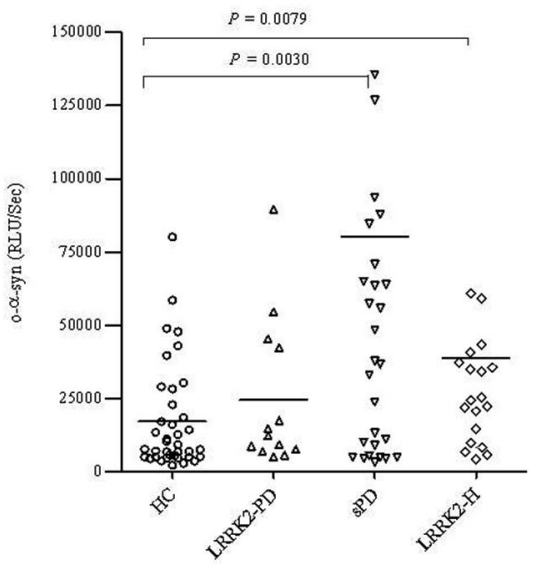

void volume (V) corresponds to $\mathrm{MW}>670 \mathrm{kDa}$. The fractions of $1 \mathrm{ml}$ which correspond to the oligomeric and monomeric $\alpha$-syn peaks were separately collected, concentrated to $100 \mu \mathrm{l}$ using a speed vac, and then analyzed by western blotting for the presence of $\alpha$-syn using anti- $\alpha$-syn (211) antibody (1:1000). (B) Scatter plot representing individual values of the levels of o- $\alpha$-syn (in relative luminescence units/second [RLU/s]) in CSF from healthy controls (HC; open circles), symptomatic PD patients with LRRK2 point mutations (LRRK2-PD; open triangles), patients with (sPD; inverted open triangles), and healthy asymptomatic LRRK2 mutation carriers (LRRK2-H; open squares). Each bar represents the mean value. 
Table 2 | Spearman correlations between CSF $\alpha$-syn species, disease duration (months), and Hoehn and Yahr stage in sPD and LRRK2-PD.

\begin{tabular}{|c|c|c|c|c|c|c|}
\hline \multicolumn{4}{|c|}{ Disease duration (months) } & \multicolumn{3}{|c|}{ Hoehn and Yahr stage } \\
\hline$t-\alpha-\operatorname{syn}$ & NS & NS & NS & NS & NS & NS \\
\hline
\end{tabular}

${ }^{*} P<0.05,{ }^{*} P<0.01,{ }^{*} * P<0.001$, NS $=$ Not-Significant.

$n=35)(P<0.001$, Mann-Whitney $\mathrm{U}$ test $)$ and LRRK2$\mathrm{H}$ group $($ mean $\pm \mathrm{SEM}=103.1 \pm 47.91, n=20)(P<$ 0.05 , Mann-Whitney $U$ test) than in the healthy control group (mean $\pm \mathrm{SEM}=40.97 \pm 21.62, n=42)$. No significant difference in the ratio of $o-\alpha$-syn to $t-\alpha$-syn was found in the LRRK2-PD relative to controls (mean $\pm \mathrm{SEM}=24.08 \pm 7.359$, $n=13)(P=0.5297$, Mann-Whitney $\mathrm{U}$ test $)$, possibly because of the small number of individuals in this group ( $n=13$ vs. $n=42)$.

Figure 4 shows the ROC curve for CSF o- $\alpha$-syn and the ratio of $o-\alpha$-syn to $t-\alpha$-syn in the discrimination of PD patients from controls. The ROC curve indicates that cutoff values of 31,671.9 RLU/s for CSF o- $\alpha$-syn and $24.1 \%$ for the CSF o- $\alpha$-syn to $t-\alpha$-syn ratio were the most reliable measures to distinguish sPD from controls. Detection of CSF $\alpha$-syn oligomers yielded a sensitivity of $65 \%$ (95\% CI, 53-77\%) and a specificity of $83 \%$ (95\% CI, 73$93 \%$ ), with an area under the curve (AUC) of 0.74 (95\% CI, 0.600.88 ). The $o-\alpha$-syn to $\mathrm{t}-\alpha$-syn ratio yielded a sensitivity of $73 \%$ (95\% CI, 62 to $84 \%$ ) and a specificity of $77 \%$ (95\% CI, 0.66-0.88), with an AUC of 0.79 (95\% CI, 0.67-0.91). Furthermore, the ROC analysis demonstrated that cutoff values of 20,566 RLU/s for CSF o- $\alpha$-syn and $9.4 \%$ for the CSF o- $\alpha$-syn to $t-\alpha$-syn ratio provided the most reliable measure to differentiate LRRK2-H from healthy controls. The sensitivity and specificity of CSF o- $\alpha$-syn to predict LRRK2-H were 63\% (95\% CI, 0.50-0.76) and 74\% (95\% CI, 0.620.86), respectively, with an AUC of 0.66 (95\% CI, 0.50 to 0.82 ). The sensitivity of the CSF oligomer to t- $\alpha$-syn ratio was $53 \%$ (95\% CI, 39-67\%), and the specificity was $81 \%$ (95\% CI, $0.70-0.92$ ) with an AUC of 0.64 (95\% CI, 0.47-0.80).

\section{DISCUSSION}

Currently the diagnosis of PD is based mainly on clinical symptoms. However, differential diagnosis from other parkinsonisms can be difficult and can lead to misdiagnosis. To date, no simple laboratory biomarker is available to detect individuals at risk for PD before most of their dopaminergic neurons have been lost.

Numerous studies have suggested that neuronal cell death may result from the formation of oligomeric species of $\alpha$-syn in the brain (Irvine et al., 2008). It has been previously shown that levels of soluble o- $\alpha$-syn are elevated in brain homogenates from patients with PD and DLB relative to normal brains (Paleologou et al., 2009). Recently, it has been reported by us and others significant higher levels of CSF o- $\alpha$-syn in PD patients compared to age-matched controls (Tokuda et al., 2010; Park et al., 2011; Sierks et al., 2011; Parnetti et al., 2014a,b). Levels of o$\alpha$-syn and the o- $\alpha$-syn to $t-\alpha$-syn ratio have also been shown to be higher in patients with mild $\mathrm{PD}$ ( $\mathrm{H} \& \mathrm{Y}$ grades 1 and
2) and patients with early PD (within 24 months after onset) relative to a control group (Tokuda et al., 2010). The aim of this study was to determine whether $0-\alpha$-syn is suitable biomarker for detecting PD at the early stages of the disease. Recently, abnormal PET changes and olfactory dysfunction were reported in LRRK2-H (Nandhagopal et al., 2008; Ruiz-Martínez et al., 2011; Saunders-Pullman et al., 2011). Therefore, healthy family members with LRRK2 mutations are an excellent population for validating surrogate biomarkers for early stages of PD. A recent study reported a lack of a statistically significant relationship between PET scan evidence of lost striatal dopaminergic function and the levels of DJ-1 and t- $\alpha$-syn in CSF from LRRK-H or LRRK-PD cases (Shi et al., 2012). In the present study, we assessed the levels of CSF o- $\alpha$-syn in symptomatic and asymptomatic LRRK2 mutation carriers and in SPD cases. We observed significantly elevated levels of CSF $\alpha$-syn oligomers in LRRK$\mathrm{H}$ relative to healthy controls (Figure $2 \mathrm{~B}$ ), which suggests that the formation of $\mathrm{o}-\alpha$-syn in the brain commences several years before LRRK2 mutation carriers experience any motor symptoms of PD. Furthermore, we also observed significantly higher o$\alpha$-syn levels in the CSF of sPD cases relative to healthy agematched controls (Figure 2B), which confirms previous findings (Tokuda et al., 2010; Park et al., 2011; Sierks et al., 2011). Unexpectedly, we did not observe any significant difference in the CSF levels of o- $\alpha$-syn between LRRK-PD patients and healthy controls, possibly because of the low number of individuals in this group ( $n=13$ vs. $n=42$ ). However, we noticed in this group that patients with mild PD ( $H \& Y$ grade $\leq 2)$ showed high levels of CSF o- $\alpha$-syn similar to those of $s$ PD patients. Conversely, patients with higher $\mathrm{H} \& \mathrm{Y}$ grades $(>2)$ had lower levels of $\alpha$-syn oligomers, which supports the hypothesis that at the early stages of the disease, high levels of toxic o- $\alpha$-syn accumulate in the brain. In support of this notion, we also found that levels of CSF o- $\alpha$-syn were inversely correlated with disease duration and H\&Y grade in LRRK2-PD and sPD cases, which confirms that CSF levels of o- $\alpha$-syn decrease with increasing PD severity (Table 2 ). Thus, we speculate that $o-\alpha$-syn is formed during the early stages of the disease prior to any major clinical manifestation.

Unified Parkinson's Disease Rating Scale is the most commonly used clinical scale to provide an efficient and flexible assessment of motor performance in PD patients and to monitor the degree of resultant disability. However, thus far, no strong linear relationship has been established between UPDRS scores and the progressive nigrostriatal degeneration in $\mathrm{PD}$, which may underlie the absence of a correlation between CSF $\alpha$-syn levels and UPDRS scores. Cerebrospinal fluid biomarkers mirror changes within the brain as an entire unit, whereas UPDRS scores primarily reflect 


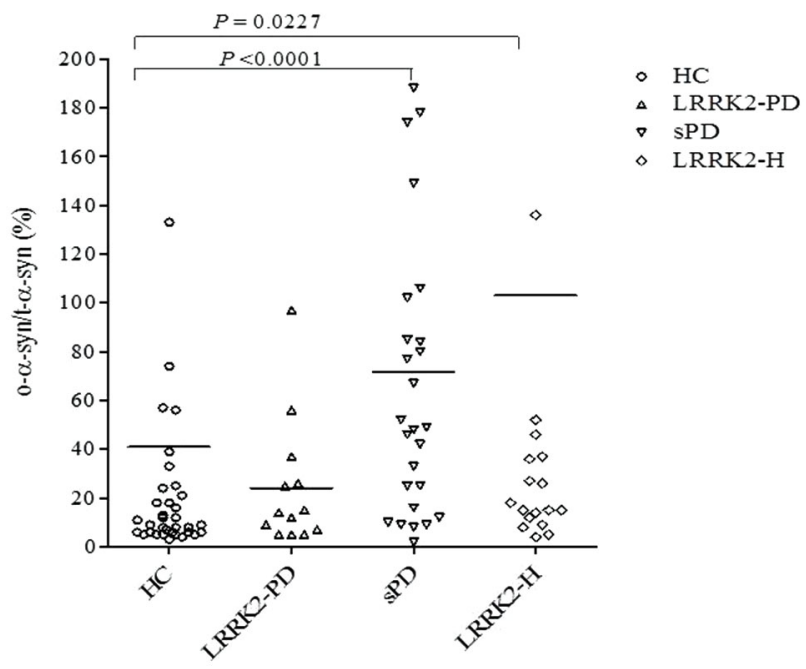

FIGURE 3 | Scatter plot presenting individual values for the ratio of $o-\alpha$-syn to $t-\alpha$-syn (o- $\alpha$-syn/t- $\alpha$-syn ratio, $\%$ ) in CSF from healthy controls (HC; open circles), symptomatic PD patients with LRRK2 point mutations (LRRK2-PD; open triangles), patients with (sPD; inverted open triangles), and healthy asymptomatic LRRK2 mutation carriers (LRRK2-H; open squares). Each bar represents the mean value. The $P$ value for each regression line is shown in each subfigure.

\section{A}

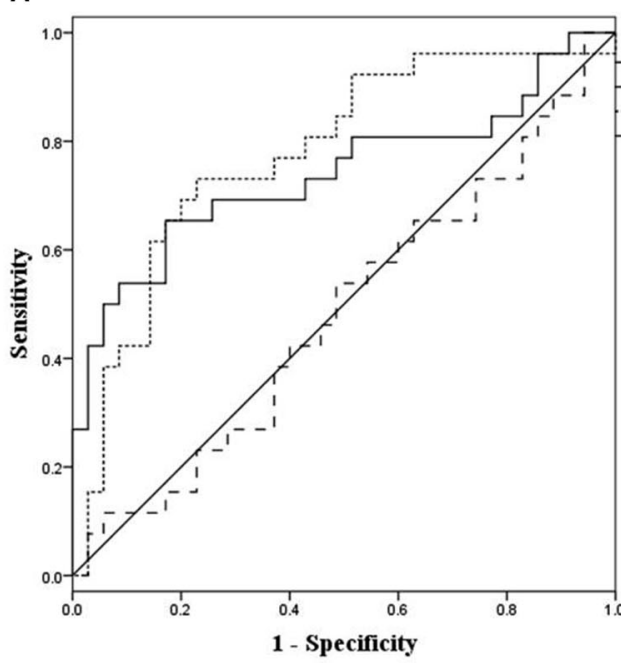

B

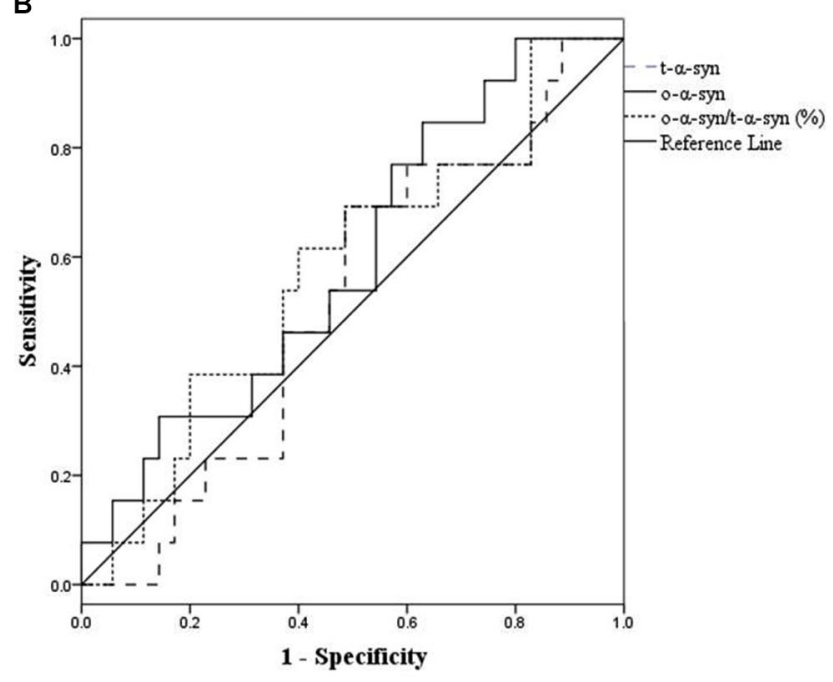

FIGURE 4 | Use of receiver operating characteristic (ROC) curves for the levels of CSF t- $\alpha$-syn, o- $\alpha$-syn and o- $\alpha$-syn/t- $\alpha$-syn ratio (\%) to discriminate between (sPD) patients and controls (A), and healthy asymptomatic LRRK2 mutation carriers (LRRK2-H) patients and controls (B).

changes in the nigrostriatal dopaminergic system. In addition, compensatory responses in PD may further confound the correlation between CSF biomarkers and the severity of PD motor symptoms (Shi et al., 2011). Moreover, DA replacement therapy provided to PD patients enhances motor function while showing little or no effect on CSF protein concentrations (Hong et al., 2010; Shi et al., 2011).

It has been recently reported that the most common LRRK2 point mutation, G2019S, initiates and enhances the formation of $\alpha$-syn aggregates (Lin et al., 2009), possibly by impairing degradation pathways such as the autophagylysosomal pathway (Ferree et al., 2012; Tong et al., 2012). Overall, the potential interactions of LRRK2 and $\alpha$-syn have not been clearly established. Although most LRRK2-related PD cases are pathologically and clinically undistinguishable from sPD, LRRK2 mutation carriers exhibit considerable clinical and pathological variability (Wider et al., 2010). Our results support the hypothesis that mutations in LRRK2 may lead to the formation of the toxic oligomeric forms of $\alpha$-syn critical for the pathogenesis of PD. However, our findings need 
to be confirmed in prospectively planned, independent cohort, particularly in samples where PD has been longitudinally assessed such as the ongoing Parkinson's Progression Markers Initiative.

In conclusion, our current pilot study suggests for the first time that quantification of $\mathrm{o}-\alpha$-syn in CSF has strong potential value as a tool for PD diagnosis and presymptomatic screening of high-risk individuals who are good candidates for clinical trials. However, large-scale, prospective, and well-controlled studies, especially those that include subjects at genetic risk, are necessary to validate the use of CSF $0-\alpha$-syn as a biomarker for PD.

\section{ACKNOWLEDGMENTS}

This study was supported by the Michael J. Fox Foundation for Parkinson's Research (NY, USA) to Omar M. A. El-Agnaf and Jan O. Aasly.

\section{REFERENCES}

Aasly, J. O., Toft, M., Fernandez-Mata, I., Kachergus, J., Hulihan, M., White, L. R., et al. (2005). Clinical features of LRRK2-associated Parkinson's disease in central Norway. Ann. Neurol. 57, 762-765. doi: 10.1002/ana.20456

Aasly, J. O., Vilariño-Güell, C., Dachsel, J. C., Webber, P. J., West, A. B., Haugarvoll, K., et al. (2010). Novel pathogenic LRRK2 p.Asn1437His substitution in familial Parkinson's disease. Mov. Disord. 25, 2156-2163. doi: 10.1002/mds.2 3265

Dächsel, J. C., and Farrer, M. J. (2010). LRRK2 and Parkinson disease. Arch. Neurol. 67, 542-547. doi: 10.1001/archneurol.2010.79

Di Fonzo, A., Rohé, C. F., Ferreira, J., Chien, H. F., Vacca, L., Stocchi, F., et al. (2005). A frequent LRRK2 gene mutation associated with autosomal dominant Parkinson's disease. Lancet 365, 412-415. doi: 10.1016/s0140-6736(05) 17829-5

El-Agnaf, O. M., Salem, S. A., Paleologou, K. E., Cooper, L. J., Fullwood, N. J., Gibson, M. J., et al. (2003). Alpha-synuclein implicated in Parkinson's disease is present in extracellular biological fluids, including human plasma. FASEB J. 17, 1945-1947. doi: 10.1096/fj.03-0098fje

El-Agnaf, O. M., Salem, S. A., Paleologou, K. E., Curran, M. D., Gibson, M. J., Court, J. A., et al. (2006). Detection of oligomeric forms of alpha-synuclein protein in human plasma as a potential biomarker for Parkinson's disease. FASEB J. 20, 419-425. doi: 10.1096/fj.03-1449com

Ferree, A., Guillily, M., Li, H., Smith, K., Takashima, A., Squillace, R., et al. (2012). Regulation of physiologic actions of LRRK2: focus on autophagy. Neurodegener. Dis. 10, 238-241. doi: 10.1159/000332599

Gilks, W. P., Abou-Sleiman, P. M., Gandhi, S., Jain, S., Singleton, A., Lees, A. J., et al. (2005). A common LRRK2 mutation in idiopathic Parkinson's disease. Lancet 365, 415-416. doi: 10.1016/s0140-6736(05)17830-1

Hong, Z., Shi, M., Chung, K. A., Quinn, J. F., Peskind, E. R., Galasko, D., et al. (2010). DJ-1 and alpha-synuclein in human cerebrospinal fluid as biomarkers of Parkinson's disease. Brain 133(Pt. 3), 713-726. doi: 10.1093/brain/ awq008

Irvine, G. B., El-Agnaf, O. M., Shankar, G. M., and Walsh, D. M. (2008). Protein aggregation in the brain: the molecular basis for Alzheimer's and Parkinson's diseases. Mol. Med. 14, 451-464. doi: 10.2119/2007-00100. Irvine

Johansen, K. K., Hasselberg, K., White, L. R., Farrer, M. J., and Aasly, J. O. (2010). Genealogical studies in LRRK2-associated Parkinson's disease in central Norway. Parkinsonism Relat. Disord. 16, 527-530. doi: 10.1016/j.parkreldis.2010. 05.005

Johansen, K. K., White, L. R., Farrer, M. J., and Aasly, J. O. (2011). Subclinical signs in LRRK2 mutation carriers. Parkinsonism Relat. Disord. 17, 528-532. doi: 10. 1016/j.parkreldis.2011.04.014

Krüger, R., Kuhn, W., Müller, T., Woitalla, D., Graeber, M., Kösel, S., et al. (1998). Ala30Pro mutation in the gene encoding alpha-synuclein in Parkinson's disease. Nat. Genet. 18, 106-108. doi: 10.1038/ng0298-106
Lin, X., Parisiadou, L., Gu, X. L., Wang, L., Shim, H., Sun, L., et al. (2009). Leucinerich repeat kinase 2 regulates the progression of neuropathology induced by Parkinson's-disease-related mutant alpha-synuclein. Neuron 64, 807-827. doi: 10.1016/j.neuron.2009.11.006

Nandhagopal, R., Mak, E., Schulzer, M., McKenzie, J., McCormick, S., Sossi, V., et al. (2008). Progression of dopaminergic dysfunction in a LRRK2 kindred: a multitracer PET study. Neurology 71, 1790-1795. doi: 10.1212/01.wnl. 0000335973.66333 .58

Nichols, W. C., Pankratz, N., Hernandez, D., Paisán-Ruíz, C., Jain, S., Halter, C. A., et al. (2005). Genetic screening for a single common LRRK2 mutation in familial Parkinson's disease. Lancet 365, 410-412. doi: 10.1016/s0140-6736(05) 17828-3

Paleologou, K. E., Kragh, C. L., Mann, D. M., Salem, S. A., Al-Shami, R., Allsop, D., et al. (2009). Detection of elevated levels of soluble alphasynuclein oligomers in post-mortem brain extracts from patients with dementia with Lewy bodies. Brain 132(Pt. 4), 1093-1101. doi: 10.1093/brain/ awn349

Park, M. J., Cheon, S. M., Bae, H. R., Kim, S. H., and Kim, J. W. (2011). Elevated levels of $\alpha$-synuclein oligomer in the cerebrospinal fluid of drug-naïve patients with Parkinson's disease. J. Clin. Neurol. 7, 215-222. doi: 10.3988/jcn.2011. 7.4.215

Parnetti, L., Chiasserini, D., Persichetti, E., Eusebi, P., Varghese, S., Qureshi, M. M., et al. (2014a). Cerebrospinal fluid lysosomal enzymes and $\alpha$ synuclein in Parkinson's disease. Mov. Disord. 29, 1019-1027. doi: 10.1002/mds. 25772

Parnetti, L., Farotti, L., Eusebi, P., Chiasserini, D., De Carlo, C., Giannandrea, D., et al. (2014b). Differential role of CSF alpha-synuclein species, tau and A $\beta 42$ in Parkinson's disease. Front. Aging Neurosci. 6:53. doi: 10.3389/fnagi.2014. 00053

Polymeropoulos, M. H., Lavedan, C., Leroy, E., Ide, S. E., Dehejia, A., Dutra, A., et al. (1997). Mutation in the alpha-synuclein gene identified in families with Parkinson's disease. Science 276, 2045-2047. doi: 10.1126/science.276.5321. 2045

Ruiz-Martínez, J., Gorostidi, A., Goyenechea, E., Alzualde, A., Poza, J. J., Rodríguez, F., et al. (2011). Olfactory deficits and cardiac 123I-MIBG in Parkinson's disease related to the LRRK2 R1441G and G2019S mutations. Mov. Disord. 26, 2026 2031. doi: $10.1002 / \mathrm{mds} .23773$

Saunders-Pullman, R., Stanley, K., Wang, C., San Luciano, M., Shanker, V., Hunt, A., et al. (2011). Olfactory dysfunction in LRRK2 G2019S mutation carriers. Neurology 77, 319-324. doi: 10.1212/WNL.0b013e318227041c

Schapira, A. H. (1999). Science, medicine and the future: Parkinson's disease. BMJ 318, 311-314. doi: 10.1136/bmj.318.7179.311

Shi, M., Bradner, J., Hancock, A. M., Chung, K. A., Quinn, J. F., Peskind, E. R., et al. (2011). Cerebrospinal fluid biomarkers for Parkinson disease diagnosis and progression. Ann. Neurol. 69, 570-580. doi: 10.1002/ana. 22311

Shi, M., Furay, A. R., Sossi, V., Aasly, J. O., Armaly, J., Wang, Y., et al. (2012). DJ1 and alphaSYN in LRRK2 CSF do not correlate with striatal dopaminergic function. Neurobiol. Aging 33, 836.e5-836.e7. doi: 10.1016/j.neurobiolaging. 2011.09.015

Sierks, M. R., Chatterjee, G., McGraw, C., Kasturirangan, S., Schulz, P., and Prasad, S. (2011). CSF levels of oligomeric alpha-synuclein and beta-amyloid as biomarkers for neurodegenerative disease. Integr. Biol. (Camb) 3, 1188-1196. doi: 10.1039/clib00018g

Singleton, A. B., Farrer, M. J., and Bonifati, V. (2013). The genetics of Parkinson's disease: progress and therapeutic implications. Mov. Disord. 28, 14-23. doi: 10. 1002/mds. 25249

Singleton, A. B., Farrer, M., Johnson, J., Singleton, A., Hague, S., Kachergus, J., et al. (2003). alpha-Synuclein locus triplication causes Parkinson's disease. Science 302:841. doi: 10.1126/science. 1090278

Sossi, V., de la Fuente-Fernández, R., Nandhagopal, R., Schulzer, M., McKenzie, J., Ruth, T. J., et al. (2010). Dopamine turnover increases in asymptomatic LRRK2 mutations carriers. Mov. Disord. 25, 2717-2723. doi: 10.1002/mds. 23356

Spillantini, M. G., Schmidt, M. L., Lee, V. M., Trojanowski, J. Q., Jakes, R., and Goedert, M. (1997). Alpha-synuclein in Lewy bodies. Nature 388, 839-840. doi: $10.1038 / 42166$

Tokuda, T., Qureshi, M. M., Ardah, M. T., Varghese, S., Shehab, S. A., Kasai, T., et al. (2010). Detection of elevated levels of $\alpha$-synuclein oligomers in CSF from 
patients with Parkinson disease. Neurology 75, 1766-1772. doi: 10.1212/WNL. 0b013e3181fd613b

Tong, Y., Giaime, E., Yamaguchi, H., Ichimura, T., Liu, Y., Si, H., et al. (2012). Loss of leucine-rich repeat kinase 2 causes age-dependent bi-phasic alterations of the autophagy pathway. Mol. Neurodegener. 7:2. doi: 10.1186/1750-1326-7-2

Wider, C., Dickson, D. W., and Wszolek, Z. K. (2010). Leucine-rich repeat kinase 2 gene-associated disease: redefining genotype-phenotype correlation. Neurodegener. Dis. 7, 175-179. doi: 10.1159/000289232

Winner, B., Jappelli, R., Maji, S. K., Desplats, P. A., Boyer, L., Aigner, S., et al. (2011). In vivo demonstration that alpha-synuclein oligomers are toxic. Proc. Natl. Acad. Sci. U S A 108, 4194-4199. doi: 10.1073/pnas.1100976108

Conflict of Interest Statement: The authors declare that the research was conducted in the absence of any commercial or financial relationships that could be construed as a potential conflict of interest.
Received: 05 June 2014; accepted: 03 September 2014; published online: 25 September 2014.

Citation: Aasly JO, Johansen KK, Brønstad G, Warø BJ, Majbour NK, Varghese S, Alzahmi F, Paleologou KE, Amer DAM, Al-Hayani A and El-Agnaf OMA (2014) Elevated levels of cerebrospinal fluid $\alpha$-synuclein oligomers in healthy asymptomatic LRRK2 mutation carriers. Front. Aging Neurosci. 6:248. doi: 10.3389/fnagi.2014.00248

This article was submitted to the journal Frontiers in Aging Neuroscience.

Copyright (๑) 2014 Aasly, Johansen, Brønstad, Warø, Majbour, Varghese, Alzahmi, Paleologou, Amer, Al-Hayani and El-Agnaf. This is an open-access article distributed under the terms of the Creative Commons Attribution License (CC BY). The use, distribution or reproduction in other forums is permitted, provided the original author(s) or licensor are credited and that the original publication in this journal is cited, in accordance with accepted academic practice. No use, distribution or reproduction is permitted which does not comply with these terms. 